\title{
ECONOMIC AND VAR SHOCKS: WHAT CAN GO WRONG?
}

\author{
Jesús Fernández-Villaverde \\ University of Pennsylvania
}

\author{
Juan F. Rubio-Ramírez \\ Federal Reserve Bank of Atlanta
}

\begin{abstract}
This paper discusses the problem of invertibility between the economic shocks in a dynamic equilibrium model and the corresponding VAR innovations. We present an algebraic check of invertibility based on the model fundamentals and we find the identification scheme that recovers the economic shocks from the VAR innovations when the model is invertible. We illustrate our results with a model of the Great Depression proposed by Christiano, Motto, and Rostagno (2003). (JEL: E00, E32, C32)
\end{abstract}

\section{Introduction}

An equilibrium of a dynamic economic model maps the history of a vector of $k$ economic shocks $w_{t}$ into a vector of $n$ observables $y_{t}, y_{t}=d\left(w^{t}\right)$, where $w^{t}$ represents the whole history of shocks $w_{t}$ up to period $t$. The economic shocks affect the fundamental elements of the theory: Preferences, technology, informational sets, government policy, measurement errors, and so on. The observables are all variables that the researcher has access to. The mapping $d(\cdot)$ is the outcome of the equilibrium behavior of the agents in the model, captured by their optimal decision rules and the consistency conditions like resource constraints and market clearing. Via the mapping $d(\cdot)$, an economic theory tightly relates shocks and observables.

Often, we are interested in dynamic models such that $d(\cdot)$ has a linear form, $y_{t}=d(L) w_{t}$, where $L$ is the lag operator. For simplicity of exposition, $w_{t}$ will be an i.i.d. normal random variable, $w_{t} \sim \mathcal{N}(0, I)$, and $y_{t}$ will have a zero mean. We call this representation of $y_{t}$ the MA representation with respect to the economic shocks. There are two ways to obtain equilibrium representations of this form. One is to compute a first-order approximation of a nonlinear model around the

Acknowledgments: We thank Tom Sargent for many comments and with whom we have worked on this research agenda. Any views expressed herein are those of the authors and not necessarily those of the Federal Reserve Bank of Atlanta or the Federal Reserve System.

E-mail addresses: Fernández-Villaverde: jesusfv@econ.upenn.edu; Rubio-Ramírez: Juan.Rubio@ atl.frb.org 
deterministic steady state. A second possibility is to derive $y_{t}=d(L) w_{t}$ as the representation of a class of dynamic models with linear transition laws and quadratic preferences.

Because $y^{t}$ is a linear function of the history of $w^{t}, y^{t}$ always "reflects" $w^{t}$. Does the history of observables "reveal" the history of economic shocks? A way to answer to the query is to check the zeros of $\operatorname{det}[d(L)]$. If the zeros are outside the unit circle, we say that $d(L)$ has an inverse that is "one-sided" in the past and present values of $y_{t}$, which implies that the Hilbert space spanned by the history of $y_{t}, H\left(y^{t}\right)$, equals the Hilbert space spanned by the history of $w_{t}$, $H\left(w^{t}\right){ }^{1}$

There is alternative approach to answer our question. Consider the VAR representation of the observables, $A(L) y_{t}=a_{t}$, with $a_{t}=y_{t}-\hat{E}\left(y_{t} \mid y^{t-1}\right)$ being the VAR innovations. We can check whether the Hilbert space spanned by the history of $a_{t}, H\left(a^{t}\right)$, equals $H\left(w^{t}\right)$. Because $a^{t}$ is construed such that $H\left(a^{t}\right)=$ $H\left(y^{t}\right)$, this approach and the one based on checking the zeros of $\operatorname{det}[d(L)]$ are equivalent. If $H\left(a^{t}\right)=H\left(w^{t}\right)$, there exists a procedure to make the impulseresponse of the VAR to match up the impulse response function of $d(L)$. If $H\left(a^{t}\right) \neq H\left(w^{t}\right)$, it is not possible to replicate $d(L)$ with the impulses responses of a VAR.

To fix ideas, consider a simple, unidimensional example. Assume that our economic model implies the following $d(L)$ :

$$
y_{t}=w_{t}+2 w_{t-1}, \quad w_{t} \sim \text { i.i.d. } N(0,1) .
$$

$\operatorname{An} \operatorname{AR}(\infty)$ of this $y_{t}$ process has a MA representation of $y_{t}$ in terms of the AR innovations:

$$
y_{t}=2\left(a_{t}+\frac{1}{2} a_{t-1}\right), \quad a_{t} \sim \text { i.i.d. } N(0,1) .
$$

The impulse-response function implied by the economic model, $(1,2,0,0, \ldots)$, is different from the impulse-response function associated with the AR representation, $(2,1,0,0, \ldots)$.

Why are the two impulse-response functions so different? First, note that $a_{t}$ belongs to the Hilbert space spanned by the history of $y_{t}$ :

$$
a_{t}=\frac{1}{2} \sum_{j=0}^{\infty}\left(-\frac{1}{2}\right)^{j} y_{t-j} .
$$

1. $H\left(x^{t}\right)$ is the Hilbert space spanned by the completion of the square summable linear combinations of current and past values of $x_{t}$. 
However, $d(L)$ is not invertible. In fact, $w_{t}$ belongs to the Hilbert space spanned by the future of $y_{t}$ :

$$
w_{t}=\frac{1}{2} \sum_{j=0}^{\infty}\left(-\frac{1}{2}\right)^{j} y_{t+j+1} \text {. }
$$

In this simple example, invertibility is determined by whether the coefficient in front of $w_{t-1}$ is bigger or smaller than one in absolute value. In general, however, dynamic models have more than one observable, more than one economic shock, and more lags. These complications expand the ways in which the observables may partially conceal the economic shocks. At the same time, more observables mean more information and more possibilities to recover the economic shocks.

To analyze the multidimensional case, we consider two recursive representations of the observables. One representation links observables to economic shocks:

$$
\begin{aligned}
x_{t+1} & =A x_{t}+B w_{t} \\
y_{t} & =C x_{t}+D w_{t},
\end{aligned}
$$

where $x_{t}$ are the states of the model and $A, B, C$, and $D$ are functions of the deep structural parameters of our model. A second representation links observables to VAR innovations:

$$
\begin{aligned}
\hat{x}_{t+1} & =A \hat{x}_{t}+K a_{t} \\
y_{t} & =C \hat{x}_{t}+a_{t},
\end{aligned}
$$

where $\hat{x}_{t}=\hat{E}\left(x_{t} \mid y^{t-1}\right)$ is the best linear predictor of $x_{t}$ and $K$ is steady-state Kalman filter gain.

Is it possible to recover the history of economic shocks $w^{t}$ from the history of VAR innovations $a^{t}$ ? The answer depends on $A, B, C$, and $D$-that is, on the structural parameters and the cross-equation restrictions implied by our economic theory. If the answer is affirmative, we have $H\left(w^{t}\right)=H\left(a^{t}\right)$. Then, because $H\left(a^{t}\right)=H\left(y^{t}\right)$, we also have $H\left(w^{t}\right)=H\left(y^{t}\right)$.

Stacking both recursive representations together:

$$
\begin{aligned}
{\left[\begin{array}{c}
x_{t+1} \\
\hat{x}_{t+1}
\end{array}\right] } & =\left[\begin{array}{cc}
A & 0 \\
K C & A-K C
\end{array}\right]\left[\begin{array}{c}
x_{t} \\
\hat{x}_{t}
\end{array}\right]+\left[\begin{array}{c}
B \\
K D
\end{array}\right] w_{t}, \\
a_{t} & =\left[\begin{array}{ll}
C & -C
\end{array}\right]\left[\begin{array}{l}
x_{t} \\
\hat{x}_{t}
\end{array}\right]+D w_{t}
\end{aligned}
$$


When $D^{-1}$ exists, ${ }^{2}$ by substituting $\left[\begin{array}{ll}x_{t} & \hat{x}_{t}\end{array}\right]^{\prime}$ into the second equation, we get the following relationship between $a_{t}$ and $w_{t}$ :

$$
G \epsilon_{t}=\left\{I+[C \quad-C]\left[I-A^{*} L\right]^{-1}\left[B D^{-1} \quad K\right]^{\prime} L\right\} D w_{t},
$$

where $a_{t}=G \epsilon_{t}$ for any $G$ matrix such that $G G^{\prime}=E a_{t} a_{t}^{\prime}$ and

$$
A^{*}=\left[\begin{array}{cc}
A & 0 \\
K C & A-K C
\end{array}\right]
$$

Therefore, the question: "Is it possible to recover the history of economic shocks $w^{t}$ from the history of VAR innovations $a^{t}$ ?" asks to check whether the zeros of

$$
\operatorname{det}\left(I+\left[\begin{array}{ll}
C & -C
\end{array}\right]\left[z I-A^{*} L\right]^{-1}\left[B D^{-1} \quad K\right]^{\prime} L\right)
$$

are inside the unit circle.

The next theorem (whose proof, like the proof of Theorem 2, can be found in Fernández-Villaverde, Rubio-Ramírez, and Sargent 2005) presents a simple algebraic check for the value of those zeros for the "square" case that $D^{-1}$ exists.

THEOREM 1. If $D^{-1}$ exists, the zeros of

$$
\operatorname{det}\left(I+\left[\begin{array}{ll}
C & -C
\end{array}\right]\left[z I-A^{*} L\right]^{-1}\left[B D^{-1} \quad K\right]^{\prime} L\right)
$$

equal the eigenvalues of $A-B D^{-1} C$ and $A$.

Theorem 1 tells us that to check if the model is invertible, we only need to compute the eigenvalues of $A-B D^{-1} C$ (because we only consider stable systems, the eigenvalues of $A$ are always less than one).

Now, let us assume that the model is invertible, that is, the eigenvalues of $A-B D^{-1} C$ are all less than one in absolute value. How do we recover $w_{t}$ from $a_{t}$ ? Theorem 2 provides us with the answer.

THEOREM 2. Suppose that $D^{-1}$ exists and that $A-B D^{-1} C$ is a stable matrix. Then in the steady state Kalman filter, $K=B D^{-1}$ and $\Sigma=E\left(x_{t}-\hat{x}_{t}\right)\left(x_{t}-\hat{x}_{t}\right)^{\prime}=$ 0 and $\hat{x}_{t}=E\left(x_{t} \mid y^{t-1}\right)$.

2. What does it mean $D^{-1}$ exist? Basically, two things. First, that we have the same number of shocks and observables. Second, that the contemporaneous effects of any two economic shocks on observables are different, that is, we do not have redundant economic shocks. 
Theorem 2 says that for a particular subset of invertible models, those with the eigenvalues of $A-B D^{-1} C$ are strictly less than one, we have that $\Sigma=0-$ that is, we can perfectly forecast the states of the model, and we can calculate the Kalman gain immediately from $K=B D^{-1}$.

How can we use Theorem 2 to recover $w_{t}$ from $a_{t}$ ? If $K=B D^{-1}$, then

$$
G \epsilon_{t}=\left\{I+[C \quad-C]\left[I-A^{*} L\right]^{-1}\left[\begin{array}{ll}
K & K
\end{array}\right]^{\prime} L\right\} D w_{t}=D w_{t} .
$$

Therefore, we need only to set $G=D$ to find $w_{t}$ using only contemporaneous $a_{t}$. The choice of $G$ is unique only up to postmultiplication by an orthogonal matrix. Also, with this identification, the impulse-response function of the VAR will match those of the economic model.

\section{A Model of the Great Depression}

To illustrate our results, we use an economy proposed by Christiano, Motto, and Rostagno (2003) (hereafter CMR). CMR present a model of the U.S. economy to analyze the role of monetary policy during the Great Depression. Because of space constraints, we only present a summary of the economy and refer the reader to the original paper for details.

A representative, perfectly competitive, firm produces a final good $Y_{t}$ at price $P_{t}$ by combining intermediate goods $Y_{j t}$ as $Y_{t}=\left(\int_{0}^{1} Y_{j t}^{1 / \lambda_{f, t}} d j\right)^{\lambda_{f, t}}$ where $1 \leq$ $\lambda_{f, t}<\infty$ is a stochastic process that controls the elasticity of substitution among intermediate goods.

Intermediate goods are produced by competitive monopolists using capital $K_{j t}$ and labor $l_{j t}$ according to the production function

$$
Y_{j t}= \begin{cases}\varepsilon_{t} K_{j t}^{\alpha}\left(z_{t} l_{j t}\right)^{1-\alpha}-\Phi z_{t} & \text { if } \varepsilon_{t} K_{j t}^{\alpha}\left(z_{t} l_{j t}\right)^{1-\alpha}>\Phi z_{t} \\ 0 & \text { otherwise }\end{cases}
$$

where $\Phi$ is a fixed cost, $\varepsilon_{t}$ is a stationary technology shock, and $z_{t}=\mu z_{t-1}$ is the trend growth rate in technology. The intermediate good producers fix their prices $P_{j t}$ subject to Calvo pricing frictions. In each period, a fraction $1-\xi_{p}$ of intermediate good producers reoptimize its price, whereas the fraction $\xi_{p}$ keeps the prices of the last period, indexed by past inflation, $\pi_{t-1}$. The rental price of capital is $\operatorname{Pr}_{t}^{k}$ and the wage $W_{t}$. The firm must finance a fraction $\psi_{k}$ of the capital payments and a fraction $\psi_{l}$ of the labor payments in advance through a working-capital loan with interest rate $R_{t}$.

Capital is produced by competitive firms that buy old capital $x_{t}$ at price $Q_{\bar{K}^{\prime}, t}$ and investment goods $I_{t}$ at price $P_{t}$ to produce new capital $x_{t+1}$, with a technology 
$x_{t+1}=x_{t}+F\left(I_{t}, I_{t-1}\right)$ that reflects the adjustment costs of investment. Because the rate of transformation between $x_{t+1}$ and $x_{t}$ is 1 , the selling price of new capital is also $Q_{\bar{K}^{\prime}, t}$. Aggregate capital evolves according to $\bar{K}_{t+1}=(1-\delta) \bar{K}_{t}+$ $F\left(I_{t}, I_{t-1}\right)$, where $\delta$ is the depreciation factor.

Capital services are provided by entrepreneurs. At the end of period $t$, an entrepreneur has a net worth $N_{t}$. With $N_{t}$ and a bank loan, the entrepreneur buys an amount of capital $\bar{K}_{t+1}$. After the purchase, the capital becomes $\omega \bar{K}_{t+1}$, where $\log \omega$ is an i.i.d. normal random variable with zero mean and (stochastic) standard deviation $\sigma_{t}$. The value of $\omega$ is known after the purchase of capital while the value $\sigma_{t}$ is known before. After observing the shock, the entrepreneur rents its capital at the level of utilization, $u_{t+1}$, to maximize its profit,

$$
\left[u_{t+1} r_{t+1}^{k}-a\left(u_{t+1}\right)\right] \omega P_{t+1} \bar{K}_{t+1},
$$

where $a\left(u_{t+1}\right) \omega \bar{K}_{t+1}$ is the convex cost of utilizing capital at rate $u_{t+1}$. After production, the entrepreneur sells undepreciated capital to capital producers, pays off debt to banks, and receives a transfer $W_{t}^{e}$. These three elements determine its new net worth $N_{t+1}$. At the end of the period, a fraction $1-\gamma_{t}$ of entrepreneurs dies, their net worth distributed among households, and a fraction $1-\gamma_{t}$ of new entrepreneurs is born. Mortality ensures that the credit market restrictions remain binding by bounding (almost surely) the distribution of capital.

A representative, perfectly competitive bank engages in two activities. First, it borrows an amount $B_{t}$ from households at a nominal rate of return $R_{t+1}^{e}$ to lend it to the entrepreneurs at rate $Z_{t+1}$. The parameters of the entrepreneur's loan are chosen to maximize its utility, subject to zero profits for the bank in each state of nature and to the requirement that $R_{t+1}^{e}$ is uncontingent in time $t+1$ shocks. If the borrower cannot pay because of a low shock $\omega$, he is declared bankrupt and all his wealth is seized by the bank. For simplicity, CMR assume that there are no costs for the first activity. Second, the bank issues deposit liabilities $D_{t}^{h}$ to households. Some of these are kept as reserves while the rest are loaned as working capital in the form of deposits $D_{t}^{f}$. The management of total deposits $D_{t}=D_{t}^{h}+D_{t}^{f}$ requires capital, $K_{t}^{b}$, labor, $l_{t}^{b}$, and excess reserves, $E_{t}^{r}$, according to the technology

$$
\frac{D_{t}}{P_{t}}=x^{b}\left(\left(K_{t}^{b}\right)^{\alpha}\left(z_{t} l_{t}^{b}\right)^{1-\alpha}\right)^{\xi_{t}}\left(\frac{E_{t}^{r}}{P_{t}}\right)^{1-\xi_{t}}
$$

where $x^{b}$ is a constant. Demand deposits pay an interest rate $R_{a t}$. Finally, even if there is a representative bank, we can define an interbank interest rate $R_{t}^{b}$ at which otherwise identical banks would lend to each other.

There is a continuum of households indexed by $i \in[0,1]$, which consume $C_{t}$, allocate their wealth between currency $M_{t}$, demand deposits, and time deposits, 
and supply specialized labor $h_{i, t}$ to maximize its utility

$$
E_{t} \sum_{l=0}^{\infty} \beta^{l}\left\{\log \left(C_{t+l}-b C_{t+l-1}\right)-\zeta_{t+1} \frac{\psi_{L}}{2} h_{i, t+l}^{2}-\Theta_{t}\right\}
$$

where $b$ generates habit persistence, $\zeta_{t+1}$ is a unit-mean leisure shock,

$$
\Theta_{t}=v_{t} \frac{\left[\left(P_{t+l} C_{t+l} / M_{t+l}\right)^{\theta_{t+l}}\left(P_{t+l} C_{t+l} / D_{t+l}^{h}\right)^{1-\theta_{t+l}}\right]^{1-\sigma_{q}}}{1-\sigma_{q}}
$$

is the utility from money and deposits, $v_{t}$ is a unit-mean liquidity shocks, and $\theta_{t}$ is a stochastic parameter that controls preferences for deposits.

At the beginning of each period, households split their money, $M_{t}^{b}$, between currency and bank deposits $A_{t}$, such that $M_{t}^{b}=M_{t}+A_{t+l}^{h}$. Because the central bank credits bank deposits with $X_{t}$ units of money, we have $D_{t}^{h}=A_{t}+X_{t}$. As income, households get wages, the interest payments in various form of loans, profits from firms, and net worth of deceased entrepreneurs. They use the resources for consumption, money accumulation, saving, and to pay a lump sum to balance the government budget.

Because the household is a monopolistic supplier of its labor, it will optimally set its wage $\bar{W}_{t}$ given Calvo's frictions, adjusting with probability $1-\xi_{w}$. Otherwise, they index the wage by past inflation and technological progress $W_{j, t}=\pi_{t-1} \mu W_{j, t-1}$. The different types of labor are aggregated in a labor composite by a representative, perfectly competitive firm with technology

$$
l^{s}=\left(\int_{0}^{1} h_{j}^{1 / \lambda_{w}} d j\right)^{\lambda_{w}}
$$

where $1 \leq \lambda_{w}<\infty$. Finally, there is government consumption $G_{t}=z_{t} g$, where $g$ is a constant and a monetary authority that sets $M_{t+1}^{b}=M_{t}^{b}\left(1+x_{t}\right)$.

There are eight exogenous shocks in the model: The monopoly power degree $\lambda_{f, t}$; productivity of bank reserves $\xi_{t}$; leisure shock $\zeta_{t}$; liquidity shocks $v_{t}$; preferences for deposits $\theta_{t}$; productivity shock $\varepsilon_{t}$; riskiness for entrepreneurs $\sigma_{t}$; and mortality rate $\gamma_{t}$. The shocks (some in levels, some transformed) follow an $\operatorname{ARMA}(1,1)$ process. The variable $x_{t}$ depends on the eight exogenous shocks through a linear feedback rule.

An equilibrium of the economy can be defined in a standard way and approximated by linearization. 


\section{Results}

CMR estimate their model using 13 variables:

$$
X_{t}=\left[\begin{array}{ccccccc}
\log \frac{N_{t+1}}{P_{t} Y_{t}} & \log \pi_{t} & \log l_{t} & R_{t}^{b} & \Delta \log Y_{t} & \log \frac{W_{t}}{P_{t} Y_{t}} & \log \frac{I_{t}}{Y_{t}} \\
& \log V_{t}^{1} & \log \frac{C_{t}}{Y_{t}} & P_{t}^{e} & \log d_{t}^{c} & \log V_{t}^{b} & \log d_{t}^{\gamma}
\end{array}\right]^{\prime},
$$

where $d_{t}^{c}$ and $d_{t}^{\gamma}$ are currency-to-demand deposit ratio and the bank reservesto-demand deposits ratio, $V_{t}^{1}$ and $V_{t}^{b}$ are the velocity of M1 and the monetary base and $P_{t}^{e}$, the external finance premium, defined as the difference between the expected cost of borrowing for an entrepreneur and the payment of the bank to households for time deposits. CMR assume that these 13 variables are measured with some, linear, normal errors.

The combination of 8 economic shocks and 13 measurement errors results in a model with 21 shocks. Our results of invertibility are interesting for the case where we have a "square" system. With too many shocks, we know that the system is non-invertible; with too few, we suffer from stochastic singularity.

To achieve a square system, we can proceed in three ways. One is to increase the number of observables. Because we want to use CMR's data set, we do not follow this route. Second, we can eliminate eight of the measurement errors and keep five of them. This alternative seems arbitrary to us. There is no compelling reason to think that some variables are observed with measurement error and some are not. Finally, the third alternative is to drop five of the observables and keep only eight of them. This strategy seems the most natural for our purposes.

But then, we face a second choice: Which eight variables to keep? One approach is to compute all different combinations of 8 variables out of 13 and study, for each and one of them, whether the resulting VAR is invertible or not. For completeness, we undertook this exercise. However, because of space considerations, we report only a summary of the results for two different VARs, an exercise that is sufficient to exemplify the method.

First, we select

$$
X_{t}^{1}=\left[\begin{array}{llllllll}
\log \pi_{t} & \log l_{t} & R_{t}^{b} & \log \frac{I_{t}}{Y_{t}} & \log V_{t}^{1} & \log \frac{C_{t}}{Y_{t}} & \log d_{t}^{c} & \log d_{t}^{\gamma}
\end{array}\right]^{\prime} .
$$

Second, we select

$$
X_{t}^{2}=\left[\begin{array}{llllllll}
\log \pi_{t} & \log l_{t} & R_{t}^{b} & \log \frac{I_{t}}{Y_{t}} & \log V_{t}^{1} & \log \frac{C_{t}}{Y_{t}} & P_{t}^{e} & \log d_{t}^{\gamma}
\end{array}\right]^{\prime} .
$$

Note how the only difference between the two sets of variables is the substitution of the (log of) currency-to-demand deposit ratio, $\log d_{t}^{c}$, a variable recording a quantity, for the external finance premium, $P_{t}^{e}$, a variable recording a price. 
We take our structural parameter values from CMR's estimates, which are a combination of maximum likelihood and calibration. With those values, we run the code kindly lent to us by Larry Christiano to compute the solution of the model and, with that solution, generate the corresponding $A, B, C$, and $D$. The $A$ and $B$ will be the same for both sets of observables while the $C$ and $D$ will differ in one row.

What happens if we apply our simple check of invertibility to those two sets of matrices? For $X_{t}^{1}$, we find that the model is invertible: The biggest eigenvalue is 0.976 . However, for $X_{t}^{2}$, the model is not invertible: The biggest eigenvalue is 1.030 .

What are the consequences of this lack of invertibility? First, the researcher cannot obtain the exact value of the states even with a time series for observables of infinite length, namely, $\Sigma$ is different from zero. Second, the variance-covariance matrix of the one-step ahead forecast from the VAR is bigger than the one from the true model. The covariance matrix of the innovations $a_{t}$ is $E a_{t} a_{t}^{\prime}=C \Sigma C^{\prime}+D D^{\prime}$, whereas the variance-covariance matrix of one-step ahead errors from the true model is $D D^{\prime}$. In the non-invertible case, because $\Sigma$ is different from zero, we have the extra quadratic term $C \Sigma C^{\prime}$. Third, the impulse response functions obtained from the model and from the VAR are different.

We finish by emphasizing two points. First, Watson (1994) explains how a researcher is most vulnerable to non-invertibility when her VAR excludes measures of important endogenous variables that depend on streams of expected future values of other variables. This is the situation in our example with the currencyto-demand deposit ratio: It offers information about quantities that help to reveal the true value of the states, something that the external finance premium, a price, does not do. Second, this example illustrates how difficult it can be to guess the invertibility result ex ante. It does not seem obvious or intuitive which of the two specifications of the VAR is invertible. Consequently, this example justifies the potential importance of our theoretical results in Section 2 as a device to help researchers check for invertibility.

\section{References}

Christiano, Larry, Roberto Motto, and Massimo Rostagno (2003). "The Great Depression and the Friedman-Schwartz Hypothesis.” Journal of Money, Credit, and Banking, 35, 11191197.

Fernández-Villaverde, Jesús, Juan F. Rubio-Ramírez, and Thomas J. Sargent (2005). “A,B,C's (and D)'s for Understanding VARs." Working paper, New York University.

Watson, Mark W. (1994). "Vector Autoregressions and Cointegration." In Handbook of Econometrics, edited by Daniel L. McFadden and Robert F. Engle. Vol. IV. Elsevier Science. 
Copyright of Journal of the European Economic Association is the property of MIT Press and its content may not be copied or emailed to multiple sites or posted to a listserv without the copyright holder's express written permission. However, users may print, download, or email articles for individual use. 